\title{
Les lipocortines
}

Les lipocortines sont des inhibiteurs de la phospholipase $\mathbf{A}_{2}$ dont la synthèse est augmentée par les glucocorticoïdes, expliquant ainsi le pouvoir anti-inflammatoire de ces hormones. Elles sont aussi les substrats de tyrosine kinases : celle associée au récepteur de l'EGF, et la p60'rc, produit de l'oncogène src. Les lipocortines constituent enfin l'une des sous-unités des calpactines, calciprotéines membranaires de fonction inconnue.

\section{Bernard Rothhut}

Chargé de recherche au Cnrs

\section{Françoise Russo-Marie Directeur de recherche à l'Inserm.}

\section{ADRESSE}

B. Rothhut, F. Russo-Marie : unité des venins, unité associée Institut Pasteur, Inserm es différents événements membranaires se produisant lors de la réaction inflammatoire et conduisant à la formation de médiateurs lipidiques (prostaglandines, leucotriènes et $\mathrm{PAF}$-acéther) impliquent toujours à un moment donné l'activation d'une enzyme membranaire, la phospholipase $A_{2}$. Cette activation d'une enzyme membranaire permet l'hydrolyse en position 2 des phospholipides présents dans la membrane et conduit à la libération d'un acide gras insaturé - habituellement l'acide arachidonique - et à la formation simultanée d'un lyso-phospholipide (phospholipide auquel manque un acide gras). Si le phospholipide cible de la phospholipase $\mathrm{A}_{2}$ est un éther de phosphatidylcholine, le lysodérivé formé est le lyso-PAF, précurseur du PAF-acéther (platelet activating factor) (figure 1). Dans les cellules inflammatoires, le signal induit par un stimulus inflammatoire est transmis par une série d'événements en cascade aboutissant à une stimulation de la phospholipase $A_{2}$, permettant ainsi la libération dans la cellule, soit de l'acide arachidonique précurseur des prostaglandines et des leucotriènes, soit du lyso-PAF précurseur du PAF-acéther. Une fois formés, ces produits sortent de la cellule et sont capables de stimuler à leur tour les membranes des cellules adjacentes, réinduisant alors l'activation de la phospholipase $\mathrm{A}_{2}$ membranaire, par l'intermédiaire de nouveaux événements de couplage. Une réaction inflammatoire simplifiée peut se concevoir comme un événement stimulant la membrane de cellules inflammatoires, le stimulus étant couplé à des systèmes effecteurs conduisant à la production de médiateurs lipidiques. Ces derniers, agissant comme des stimuli par eux-mêmes, amplifient le phénomène, rendant la réaction inflammatoire assez semblable à une réaction nucléaire où le réacteur serait la phospholipase $A_{2}$ et l'énergie, apportée par les phospholipides membranaires, toujours renouvelés par de puissants systèmes de réacylation. Si la phospholipase $\mathrm{A}_{2}$ était seule, cette réaction ne s'arrêterait pas et pourrait devenir dangereuse dans l'environnement biologique où elle se déroule. On conçoit donc aisément qu'il existe des signaux biologiques naturels capables de contrổler cette réaction quand elle se produit.

La découverte de la lipocortine, un inhibiteur endogène naturel de la phospholipase $\mathrm{A}_{2}$ a encore mis l'accent sur le rôle central de cette dernière dans la réaction inflammatoire ; en effet, cette découverte a été liée à celle du mécanisme d'action moléculaire et cellulaire des corticostéroïdes, qui inhibent 
l'activité de la phospholipase $\mathrm{A}_{2}$ par l'intermédiaire de l'augmentation de la synthèse de lipocortine (figure 2, page suivante).

Dans cette revue, nous discuterons comment la lipocortine a été découverte, et comment, depuis que sa séquence est élucidée, les rôles réels de la lipocortine et de la phospholipase $\mathrm{A}_{2}$ peuvent dépasser de beaucoup leurs fonctions connues dans la réaction inflammatoire. De plus, il n'existe pas une lipocortine mais une famille de lipocortines, probablement dérivées d'un gène commun ancestral, très ancien dans l'évolution.

\section{Découverte} de la lipocortine

La découverte initiale de la lipocortine a été liée à l'étude du mécanisme d'action des stéroïdes anti-inflammatoires. L'importance dévolue à l'acide arachidonique et à ses dérivés fut la conséquence de la découverte du mécanisme d'action des anti-inflammatoires non stéroïdiens, anti-inflammatoires parce qu'ils étaient capables d'inhiber l'enzyme de formation des prostaglandines, la cyclooxygénase. Depuis cette découverte, on a démontré que tous les médicaments anti-inflammatoires interferent plus ou moins avec la cascade de l'acide arachidonique. Néanmoins, les stéroïdes antiinflammatoires n'agissent pas comme les anti-inflammatoires non stéroïdiens, bien qu'ils soient capables, comme ces derniers, d'inhiber la formation des prostaglandines. Des travaux préliminaires avaient démontré que les stéroïdes interféraient avec la cascade de l'acide arachidonique en inhibant sa libération des réserves membranaires phospholipidiques : ils induisent la synthèse d'un inhibiteur protéique de la phospholipase $\mathrm{A}_{2}$ (voir figure 1).

Cette protéine antiphospholipasique a été ainsi décrite dans trois systèmes différents. Flower et $\mathrm{Di}$ Rosa [1] ont pu démontrer sa présence dans des macrophages péritonéaux de rats traités par les glucocorticostéroïdes et l'ont appelée

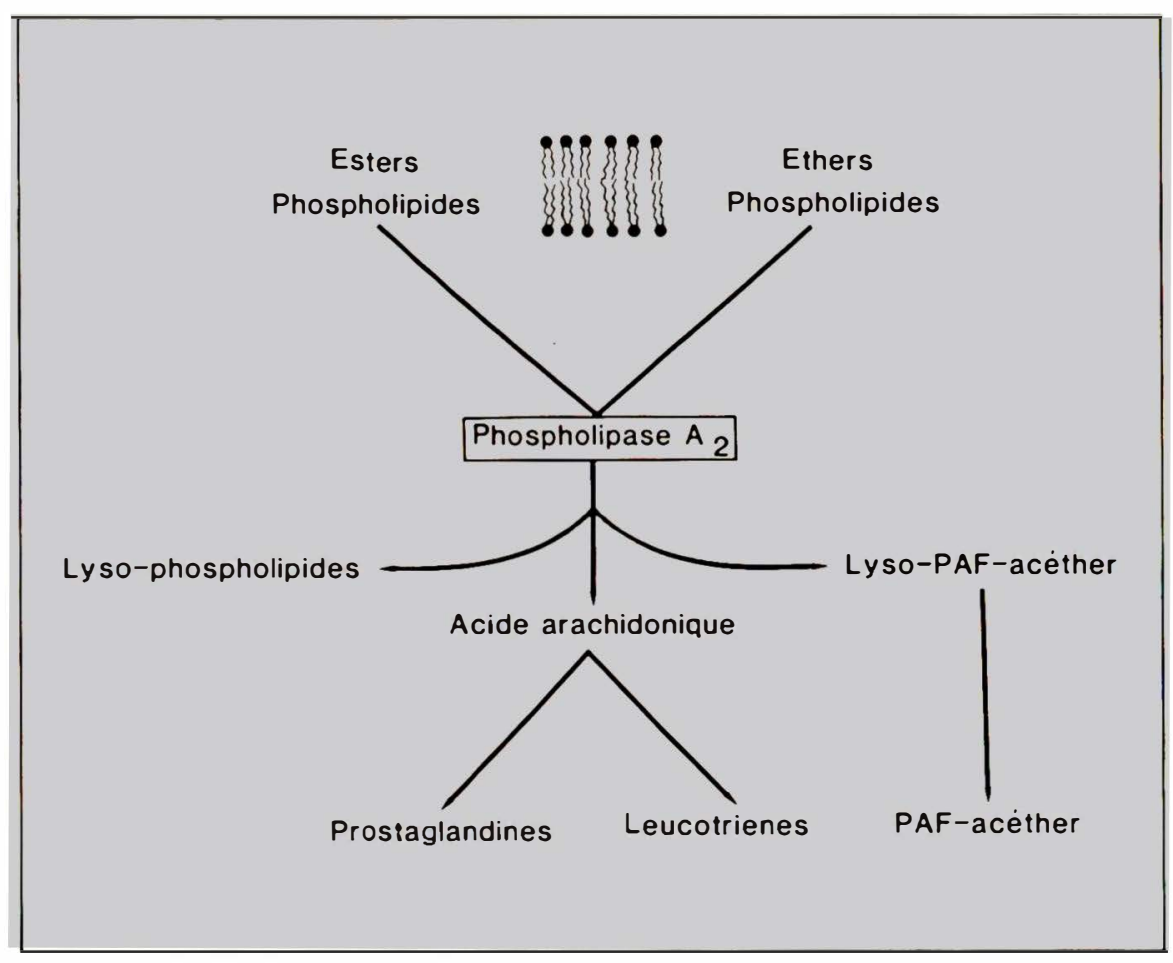

Figure 1 Rôle central de la phospholipase $A_{2}$, enzyme responsable de la formation de dérivés impliqués dans la réaction inflammatoire. lipidique représentative de la membrane plasmique.

" macrocortine "; Hirata [2] a pu démontrer le même phénomène dans les neutrophiles péritonéaux de lapin et a donné le nom de "lipomoduline ", et nous-mêmes avons démontré un phénomène semblable dans des cellules médullaires de rein de rat en culture, et avons proposé le nom de " rénocortine " [3]. Un travail fait en commun a permis de conclure qu'il s'agissait de la même protéine, puisque ces molécules étaient immunologiquement identiques, possédaient les mêmes propriétés biologiques (inhibition de la phospholipase $A_{2}$ ), la même régulation biologique (induction par les glucocorticoïdes) et les mêmes caractéristiques biochimiques (un poids moléculaire principal de $40 \mathrm{kd}^{*}$ et la propriété d'être clivé en fragments protéoly-

- $k d$ = kilodalton, unité de poids moléculaire. $1 \mathrm{kd}$ $=1000$ de poids moléculaire. tiques actifs de 30 et $15 \mathrm{kd}$ ). Le nom commun de "lipocortine " a alors été adopté [4].

\section{Une expression constitutive}

Si la lipocortine a été découverte par le fait qu'elle est sous le contrôle génomique des glucocorticoïdes, de nombreuses questions demeurent encore sans réponse, par exemple : comment inhibe-telle la phospholipase $\mathrm{A}_{2}$ ? Existe$t$-il une lipocortine endogène dans les cellules et, dans l'affirmative, comment les glucocorticoïdes interferent-ils avec elle ? Enfin, la lipocortine nouvellement formée, inhibe-t-elle la phospholipase $\mathrm{A}_{2}$ de l'intérieur ou de l'extérieur de la cellule, problème d'autant plus difficile à résoudre que la phospholipase $\mathrm{A}_{2}$ cellulaire n'est pas encore aujourd'hui biochimiquement identifiée.

Les travaux préliminaires menés 


\section{RÉFÉRENCES}

1. Blackwell GJ, Carnuccio R, DiRosa M, Flower RJ, Parente L, Persico P. Macrocortin. A polypeptide causing the antiphospholipase like effects of glucocorticoids. Nature 1980 ; $287: 147-9$

2. Hirata F, Schiffmann D, Venkatasubramanian K, Salomon D, Axelrod J. A phospholipase A2 inhibitory protein in rabbit neutrophils induced by glucocorticosteroids. Proc Natl Acad Sci USA 1980 ; 77 : 2533-6.

3. Cloix JF, Colard O, Rothhut B, RussoMarie F. Characterization and partial purification of " renocortins ". Two polypeptides formed in renal cells causing the antiphospholipase like action of glucocorticoids. BrJ Pharmacol $1983 ; 79: 312-21$.

4. DiRosa M, Flower RJ, Hirata F, Parente L, Russo-Marie F. Lipocortins, nomenclature announcement. Prostaglandins $1984 ; 28: 441-4$.

5. Hirata F. The regulation of lipomodulin, a phospholipase inhibitory protein, in rabbit neutrophils by phosphorylation. $J$ Biol Chem $1981 ; 256: 7730-3$.

6. Rothhut B, Russo-Marie F, Cousin M, Lando D. Renocortin. A phospholipase A2 inhibitory protein induced by antiinflammatory steroids : pharmacological and biological properties, development of monoclonal antibodies. In : Tumer P, Paton W, Mitchell JF, eds. Proceedings of the 9th IUPHAR Congress of Pharmacology. London : Macmillan

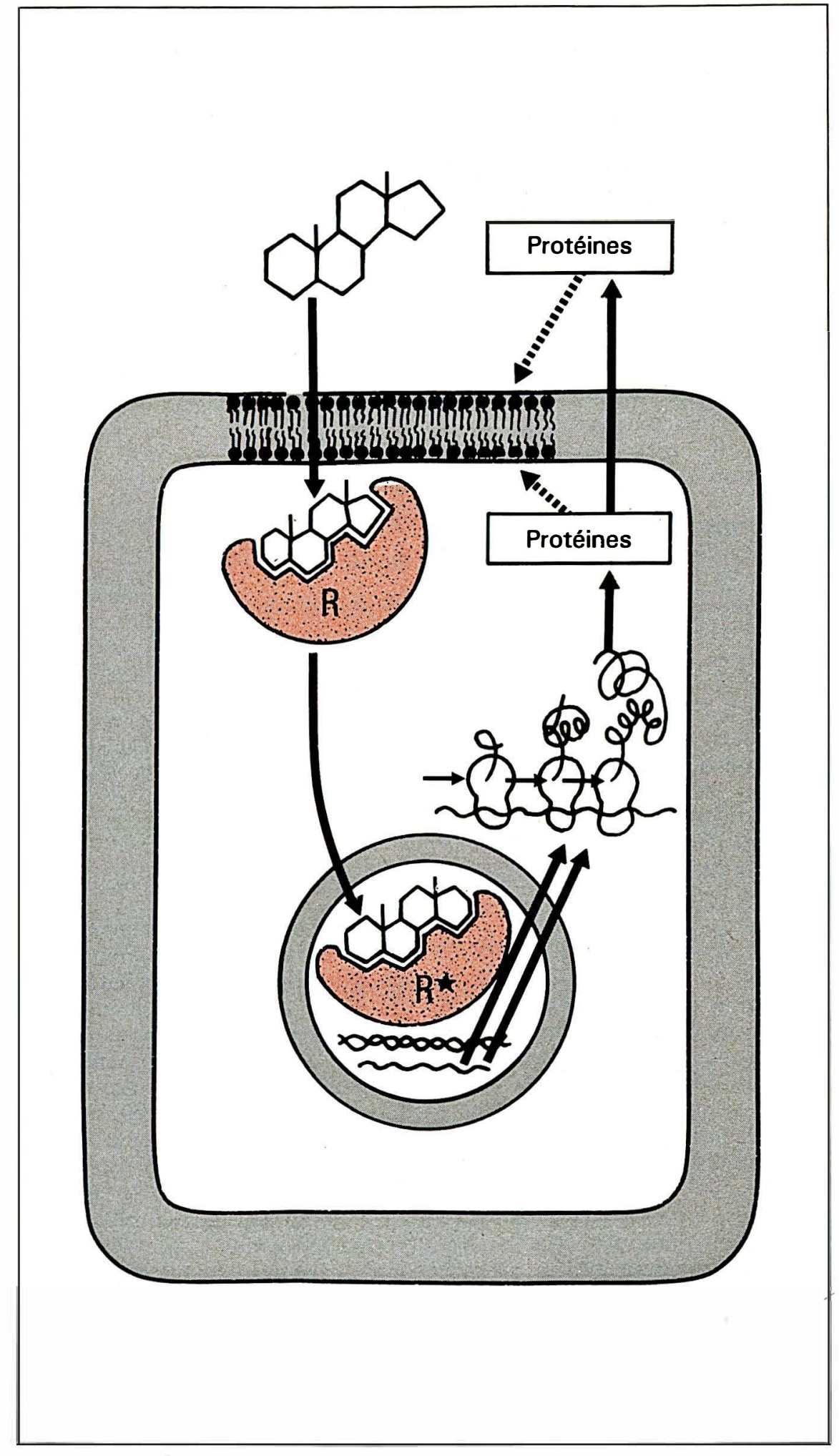

Figure 2. Schéma du mode d'action des glucocorticostéroïdes. Les stéroïdes entrent dans la cellule par diffusion passive et se lient à un récepteur spécifique. Le complexe va ensuite interagir avec I' ADN de la cellule pour induire une transcription en ARN messager qui va être traduit en protéines. $\mathrm{Ce}$ sont ces protéines qui vont être responsables de l'effet biologique induit par les hormones. 


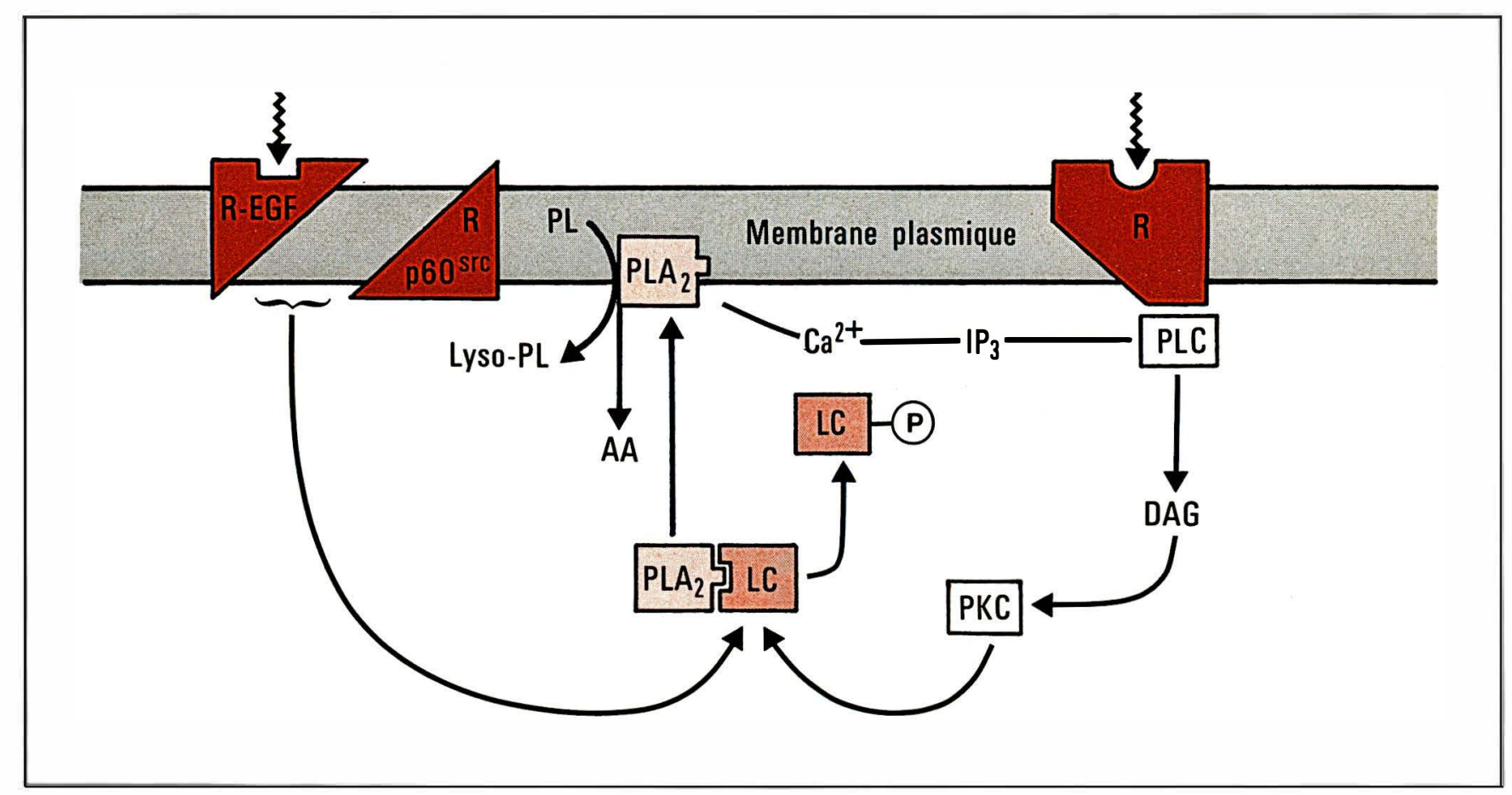

Figure 3. Schéma spéculatif du mode d'action et de la régulation des lipocortines au cours de l'activation cellulaire. R-EGF : récepteur de l'" epidermal growth factor "; 660 src : protéine p60src; PLA $A_{2}:$ phospholipase $A_{2}$; LC et LC-P : lipocortine et lipocortine phosphorylée; PL : phospholipide; Lyso-PL : Lyso-phospholipide ; AA : acide arachidonique; PLC : phospholipase C; DAG : diacylglycérol ; IP3 : inositol triphosphate ; PKC : protéine kinase C ; $\mathrm{Ca}^{2+}$ : calcium. La partie droite du schéma montre l'activation de la phospholipase $C$ après stimulation d'un récepteur cellulaire entraînant l'hydrolyse des polyphosphoinositides en diacylglycérol (activateur de la protéine kinase C) et en inositol triphosphate (mobilisateur de calcium). La protéine kinase C ainsi activée va phosphoryler la lipocortine soit directement au niveau de résidus sérines et/ou thréonines, soit par l'intermédiaire de la phosphorylation d'une protéine tyrosine-kinase. La phosphorylation de la lipocortine entraîne son inactivation vis-à-vis de la phospholipase $A_{2}$. Une augmentation simultanée de la concentration en calcium va permettre à l'enzyme d'hydrolyser les phospholipides membranaires et générer ainsi des médiateurs capables d'amplifier la réaction en restimulant des récepteurs externes. La partie gauche du schéma montre que les lipocortines (I et II) peuvent servir de substrat respectivement à la tyrosine kinase du récepteur à l'EGF et à la protéine kinase p60src, permettant à nouveau à la phospholipase $A_{2}$ de devenir pleinement active.

par les différents groupes cités cidessus ont démontré la présence de lipocortine après un traitement par les glucocorticoïdes, dans le surnageant des cellules ou dans le liquide péritonéal, indiquant que la lipocortine n'était pas seulement synthétisée mais aussi excrétée par les cellules. Néanmoins, Hirata avait rapporté la présence d'une protéine identique à la lipocortine à l'intérieur des cellules [5]. Utilisant un anticorps monoclonal dirigé contre la lipocortine de rein de rat, notre groupe [6] avait trouvé la présence de la protéine aussi bien dans les cellules témoins que dans les cellules traitées par les corticostéroïdes, démontrant qu'il existait une lipocortine endogène. Par les techniques de détection que nous avions utilisées, nous n'avions pu mettre en évidence de différence significative entre les deux types de cellules, suggérant que si les corticoïdes induisaient la synthèse de lipocortine, celle-ci survenait sur une forte concentration endogène préexistante. De plus, il n'est pas exclu qu'ils induisent la synthèse d'une protéine un peu différente, possédant une très forte activité antiphospholipasique $A_{2}$.

\section{Régulation par phosphorylation}

Hirata avait déjà démontré en 1981 que l'activité de la lipocortine pouvait être régulée par phosphorylation; la lipocortine une fois phosphorylée perd sa capacité à inhiber la phospholipase $\mathrm{A}_{2}$ [5]. De plus, une régulation de l'activité de la lipocortine par la protéine kinase $\mathrm{C}$ a été démontrée dans les neutrophiles de lapin [5], et par la tyrosine kinase dans les thymocytes murins [7]. Notre groupe a rapporté la présence de lipocortine dans les pla- 


\section{RÉFÉRENCES}

7. Hirata F, Matsuda K, Notsu Y, Hattori T, DelCarmine R. Phosphorylation of a tyrosine residue of lipomodulin in mitogen-stimulated murine thymocytes. Proc Natl Acad Sci USA $1984 ; 81: 4717-21$.

8. Touqui L, Rothhut B, Shaw A, Fradin A, Vargaftig BB, Russo-Marie F. Platelet activation - a role for a $40 \mathrm{~K}$ antiphospholipase A2 protein indistinguishable from lipocortin. Nature 1986 ; 321 : 177-80.

9. Blake-Pepinsky R, Sinclair LK, Browning $\mathrm{JL}$, et al. Purification and partial sequence analysis of a $37 \mathrm{KDa}$ protein that inhibits phospholipase A2 activity from rat peritoneal exudates. J Biol Chem 1986 ; 261 : 4246-9.

10. Wallner BP, Mattaliano RJ, Hession C et al. : Cloning and expression of human lipocortin, a phospholipase A2 inhibitor with potential anti-inflammatory activity. Nature 1986 ; 320 : 77-80.

11. Blake-Pepinsky R, Sinclair LK. Epidermal growth factor-dependent phosphorylation of lipocortin. Nature $1986 ; 321$ : 81-84.

12. Huang $\mathrm{K}-\mathrm{S}$, Wallner BP, Mattaliano $\mathrm{RJ}$, Browning JL, et al. Two human $35 \mathrm{KD}$ inhibitors of phospholipase A2 are related to substrates of $\mathrm{p} 60$ src and of epidermal growth factor receptor kinase. Cell 1986 ; 46 : 191-9.

13. De BK, Misono KS, Lukas TJ, Mroczkowski B, Cohen S. A calcium-dependent 35-kilodalton substrate for epidermal growth factor receptor/kinase isolated from normal tissue. $J$ Biol Chem 1986 ; 261 : 13784-92.

14. Glenney JR, Jr. Two related but different forms of the $36,000 \mathrm{Mr}$ tyrosine kinase substrate (calpactins) which interact with phospholipid and actin in a $\mathrm{Ca}^{2}+$-dependent manner. Proc Natl Acad Sci USA 1986; 83 : 4258-62.

15. Kristensen $T$, Saris CJM, Hunter $T$, et al. Primary structure of bovine calpactin I heavy chain ( $\mathrm{p} 36$ ), a major cellular substrate for retroviral protein-tyrosine kinases : homology with the human phospholipase A2 inhibitor lipocortin. Biochemistry $1986 ; 25$ : 4497-503.

16. Saris CJM, Tack BF, Kristensen T, Glenney JR, Hunter T. The cDNA sequence for the protein tyrosine kinase substrate p36 (Calpactin I heavy chain) reveals a multidomain protein with internal repeats. Cell $1986 ; 46$ : 201-12.

17. Geisow MJ, Fritsche U, Hexham HM, Dash B, Johnson T. A consensus amino-acid repeat in Torpedo and mammalian $\mathrm{Ca}^{2}+$ dépendent membrane-binding proteins. Nature 1986 ; 320 : 636-8.

18. Geisow MJ. Common domain structures of $\mathrm{Ca}^{2}+$ and lipid-binding proteins. FEBS Lett quettes. Dans ces cellules, l'activité de la lipocortine est modifiée par la thrombine et par les phorbolesters, impliquant donc une activation de la protéine kinase $C$ [8]. Nous avons pu démontrer en outre que la phosphorylation de la lipocortine par la thrombine ou les phorbol-esters contrôlait l'activité endogène de la phospholipase $A_{2}$. Ces résultats nous ont permis de proposer un schéma de l'activation membranaire (figure 3, page précédente) prenant en compte à la fois la phopholipase $\mathrm{A}_{2}$ et la lipocortine : l'activation d'un récepteur membranaire par un ligand conduirait à une stimulation de la phospholipase $\mathrm{C}$ entraînant la coupure des polyphosphoinositides en diacylglycérol (DG) et inositol triphosphate $\left(\mathrm{IP}_{3}\right)$. Le diacylglycérol stimule la protéine kinase $\mathrm{C}$, qui va phosphoryler diverses protéines parmi lesquelles la lipocortine. Une fois phosphorylée, la lipocortine n'est plus capable d'inhiber la phospholipase $A_{2}$. Néanmoins, cette seule modification n'est pas suffisante : un second événement provoqué par l'inositol triphosphate, une mobilisation du calcium, est nécessaire. Le calcium va alors stimuler la phospholipase $A_{2}$, libérée de son inhibiteur naturel, la lipocortine. Des résultats récents obtenus dans notre laboratoire sur les effets du phorbol myristate acétate (stimulateur comme le diacylglycérol de la protéine kinase C) et de l'ionophore A23187 (possédant, tel l' $\mathrm{IP}_{3}$, la capacité de modifier le calcium endocellulaire) nous ont permis de confirmer la validité d'un tel schéma de régulation dans la plaquette.

\section{Sites multiples de phosphorylation}

La lipocortine a été récemment séquencée [9], son $\mathrm{ADN}_{\mathrm{c}}$ a été cloné et exprimé dans $E$. coli par Wallner et al. [10] (Biogen, Boston). On a pu démontrer par la technique de Northern-blot* que, même en l'absence de glucocorti-

\footnotetext{
- Northern-blot : voir lexique $\mathrm{m} / \mathrm{s}, n^{\circ} 9$, vol. 2 , p. 518 .
}

coïdes, le messager de la lipocortine était présent dans de nombreuses cellules, principalement dans les macrophages, le poumon, le thymus, la rate et, à un moindre degré, dans le foie, le cœur, le cerveau, les muscles et le rein, confirmant la présence d'un taux de lipocortine endogène de base. De plus, le groupe de Biogen a pu démontrer la présence de lipocortine dans certaines lignées cellulaires en culture et son absence dans d'autres, telles des lignées de cellules lymphoïdes ou certaines lignées de cellules nerveuses. Comme cela avait été proposé par Hirata et al. [5, 7] et par Touqui et al. [8], la lipocortine possède en effet des sites de phosphorylation sur une tyrosine et sur une sérine. Dans la lignée cellulaire A431, la lipocortine peut être phosphorylée par l'activité tyrosine kinase du récepteur pour l'EGF (Epidermal Growth Factor) [11]. Cette lipocortine, d'un poids moléculaire de $35 \mathrm{kd}$, a été appelée lipocortine $I^{* *}$.

\section{Autres lipocortines}

Le groupe Biogen a non seulement cloné et séquencé l'ADN de la lipocortine I, mais aussi purifié à partir du placenta humain, deux protéines différentes, les lipocortines I et II [12]. Ces deux protéines possèdent les mêmes propriétés biologiques anti-phospholipasiques $A_{2}$ mais n'ont que $50 \%$ d'homologie de séquence. De plus, l'analyse peptidique, les études immunologiques et la détermination de la séquence protéique complète ont démontré que la lipocortine I, le substrat de la tyrosine kinase du récepteur de l'EGF des cellules A 431, et la calpactine II étaient des protéines voisines ayant des séquences à plus de $80 \%$ homologues [13, 14]. Des études similaires ont révélé que la lipocortine II était un analogue humain de la protéine p36, substrat du produit p60 ${ }^{\text {src }}$ des oncogènes cellulaires et viraux c- et vsrc. Cette protéine p36 avait été

* Voir nouvelle m/s, $n^{\circ} 9$, vol. 2, p. 522. 
déjà caractérisée dans les fibroblastes d'embryon de poulet et dans les préparations de la bordure en brosse de l'intestin de bœuf, et l'on avait montré qu'elle était identique à la chaîne lourde de la calpactine I $[15,16]$. La calpactine est un hétérotétramère abondant dans la bordure en brosse des entérocytes et composé de deux sous-unités p36 et de deux sous-unités plus légères, p10 (10 000 de poids moléculaire). En présence de calcium, le tétramère se lie à l'actine et à la spectrine des cellules non érythroïdes (deux protéines du cytosquelette de la cellule) ; toujours en présence du calcium, la calpactine a également une très forte affinité pour les phospholipides, le complexe calpactine-calcium-phospholipides ayant une plus grande affinité pour l'actine que la protéine fixant du calcium seule. La spectrine étant localisée à la face interne des membranes plasmiques, la calpactine a une localisation principalement membranaire. Il existe en fait tout une famille de protéines liant le calcium et capable de se fixer aux biomembranes, protéines qui semblent extrêmement conservées au cours de l'évolution: des anticorps développés contre la calélectrine, calciprotéine du poisson torpille " torpedo marmorata", reconnaissent dans les tissus de mammiferes des protéines $\mathrm{p} 7 \mathrm{C}$, p36 et p32.5 [17]. La p36 (et peut-être la p32.5) semblent bien être la lipocortine II, c'est-à-dire la chaîne lourde de la calpactine [18].

\section{Conclusion}

Ces différents résultats amènent à se poser la question du rôle réel des lipocortines : comment interviennent-elles dans la réaction inflammatoire, possèdent-elles d'autres propriétés que celle d'inhiber la phospholipase $A_{2}$, et surtout, comment peuvent-elles être à la fois présentes dans les cellules comme un constituant endogène de ces dernières et sécrétées sous l'influence des glucocorticoïdes alors qu'il n'existe pas de séquence peptide signal dans le gène des lipocortines ? Nous proposons actuellement l'existence d'une ou plusieurs lipocortines endocellulaires dont la fonction principale serait d'inhiber la phospholipase $\mathrm{A}_{2}$ endocellulaire. En effet, il est connu que dans des conditions de repos, l'enzyme est inactive. En revanche, l'arrivée d'un stimulus inflammatoire sur les récepteurs spécifiques de la membrane cellulaire déclenche, via divers systèmes de couplage encore mal connus, une cascade d'événements comportant : activation de la protéine kinase $\mathrm{C}$ et de tyrosine kinases, phosphorylation des lipocortines, activation de la phospholipase $\mathrm{A}_{2}$ et production de lipides pharmacologiquement actifs, intermédiaires de l'inflammation (figure 3).

Ces derniers sont capables de réinitier le mécanisme en interagissant avec des récepteurs de membrane d'autres cellules. Nous proposons que les corticostéroïdes, en augmentant le nombre des molécules de lipocortine capables d'inhiber la phospholipase $\mathrm{A}_{2}$, vont freiner le mécanisme en supprimant la transmission du stimulus vers les systèmes effecteurs. Ce schéma est trop simple et ne propose qu'une explication très partielle du rôle de la lipocortine et ne permet pas de répondre à toutes les questions posées par la découverte de cette protéine. En effet, pourquoi existe-t-il autant de protéines similaires à la lipocortine, et si tel est le cas, pourquoi ces différentes protéines sontelles capables d'inhiber la phospholipase $A_{2}$, de lier le calcium et de se fixer aux biomembranes ? La fonction antiphospholipasique $\mathrm{A}_{2}$ est-elle la seule importante ? ou existe-t-il d'autres fonctions de ces protéines, liées par exemple à l'induction d'une modification non spécifique des phospholipides membranaires qui pourrait jouer un rôle dans la différenciation cellulaire ? De plus comment les lipocortines sont-elles phosphorylées sur des sites tyrosine et sérine, parallèlement et/ou séquentiellement?

\section{Summary}

Lipocortins belong to a family of proteins whose major function is to inhibit phospholipase $A_{2}$. Phospholipase $A_{2}$ is a membrane enzyme activated by various inflammatory stimuli leading to the cleavage of membrane phospholipids and giving rise to prostaglandins, leukotrienes and PAF-acether, all lipid-derived mediators of the inflammatory reaction. These mediators leave the cell where they have been formed, trigger the neighbouring cell membrane and reinitiate a succession of events leading to the reactivation of phospholipase $\mathrm{A}_{2}$. Lipocortins are endocellular proteins, able to prevent phospholipase $\mathrm{A}_{2}$ from cleaving phospholipids, inhibiting therefore stimu us-response coupling. Glucocorticosteroids induce the synthesis of lipocortins which are responsible of most of the anti-inflammatory properties of these hormones. The lipocortins' activities are regulated by phosphorylation implicating protein kinase $\mathrm{C}$ and tyrosine kinase. Lipocortins are substrates of tyrosine kinases : of the EGF receptor, and of the p60 src, a src oncogene product. Finally, lipocortins are similar to calpactins and to a family of ca cium and phospholipid binding proteins whose biological functions are not known.

\section{TIRÉS À PART}

B. Rothhut, F. Russo-Marie : unité des venins, unité associée Institut Pasteur, Inserm U. 285, 25 rue du Dr-Roux, 75015 Paris. 